\title{
Pu-Ga Standards for Microanalysis and Matrix Correction Development
}

Charles C. Davis, Rollin E. Lakis

University of California, Los Alamos National Laboratory, Nuclear Materials Division, Mailstop E 574, P.O. Box 1663, Los Alamos NM 87545.

Plutonium $(\mathrm{Pu})$, atomic number of 94, is known to have 6 allotropes (crystalline forms) at atmospheric pressure between room temperature and $640^{\circ} \mathrm{C}$, the melting point of $\mathrm{Pu}$. The alphaphase (monoclinic) at room temperature is hard and brittle making it a less than idealmetal for various metallurgical processes. A number of elements, including gallium $(\mathrm{Ga})$, may be added to $\mathrm{Pu}$ to stabilize the FCC delta-phase at room temperature. This phase is ductile, and a much more suitable material for the manufacture of complex parts. During the solidification of Pu-Ga alloys (nominal $1 \mathrm{wt} \% \mathrm{Ga}$ ) from the melt, the first delta-phase material is formed Ga-rich. This is due to the shape of the delta-epsilon phase field and rapid Ga diffusion at this temperature. The resulting microstructure is heavily cored with high Ga concentrations in the center of every grain and decreasing concentrations near grain boundaries. The extent of Ga segregation can be affected by controlling the cooling rate through the delta-epsilon phase field and by homogenizing the alloy in the high temperature range of the delta phase field. It is important that the Ga be relatively homogenous to ensure that during subsequent processing all the material remains stabilized in the delta phase, and does not transform to alpha-phase under the influence of stress and temperature.

Some barriers to accurate analysis of $\mathrm{Pu}$ containing materials include: control of radioactive contamination during specimen preparation and microscopy; pure Pu metal is very reactive (pyrophoric) and is not a suitable microanalysis standard; matrix correction procedures were developed around the transition metals not the actinides. To address the two latter concerns, an investigation is underway to improve the accuracy of $\mathrm{Pu}$ microanalysis through an increased understanding of the performance of matrix correction algorithms on actinidebased materials.

Six Pu-Ga alloys were prepared with Ga concentrations between $0.5 \mathrm{wt} \%$ and $2.0 \mathrm{wt} \%$. The alloys were prepared by arc melting and homogenized by repeated heat treatments and mechanically rolling. The alloy standards were mounted in epoxy and mechanically polished. The intensity of Ga K-alpha x-rays $(9.25 \mathrm{keV})$ were measured by wavelength spectrometry using a LIF (200) diffracting crystal.

The top graph on the following page is a calibration curve construction. The solid line represents the experimental measurements. The statistical counting error and standard deviation from multiple measurements are smaller than the square data markers. The dottedline represents the intensity calculated by ZAF (John Armstrong's TRYZAF, Duncumb \& Reed Z, Philibert A) k ratios and placed on the graph using a pure Ga metal standard count rate. From this comparison, it is observed that ZAF systematically inflates the concentration of $\mathrm{Ga}$ by $42 \%, 37 \%$, and $35 \%$ at $\mathrm{Ga}$ weight fractions of $0.005,0.01$, and 0.015 respectively. There are a number of possible explanations related to the selection and origin of the various constants used in the ZAF construction (mean ionization potential, stopping power, ect.). Understanding the deviation of ZAF results from standard measurements and the improvement of the matrix correction is the focus of continuing efforts.

The bottom graph is presented as an Ziebold, Ogilvie atfactor analysis where the slope of the data equals one minus the a-factor. The nonlinear nature of the measured and calculated data sets suggests that perhaps a polynomial a-factor approach may be successful if the systematic inaccuracies of ZAF cannot be corrected. 

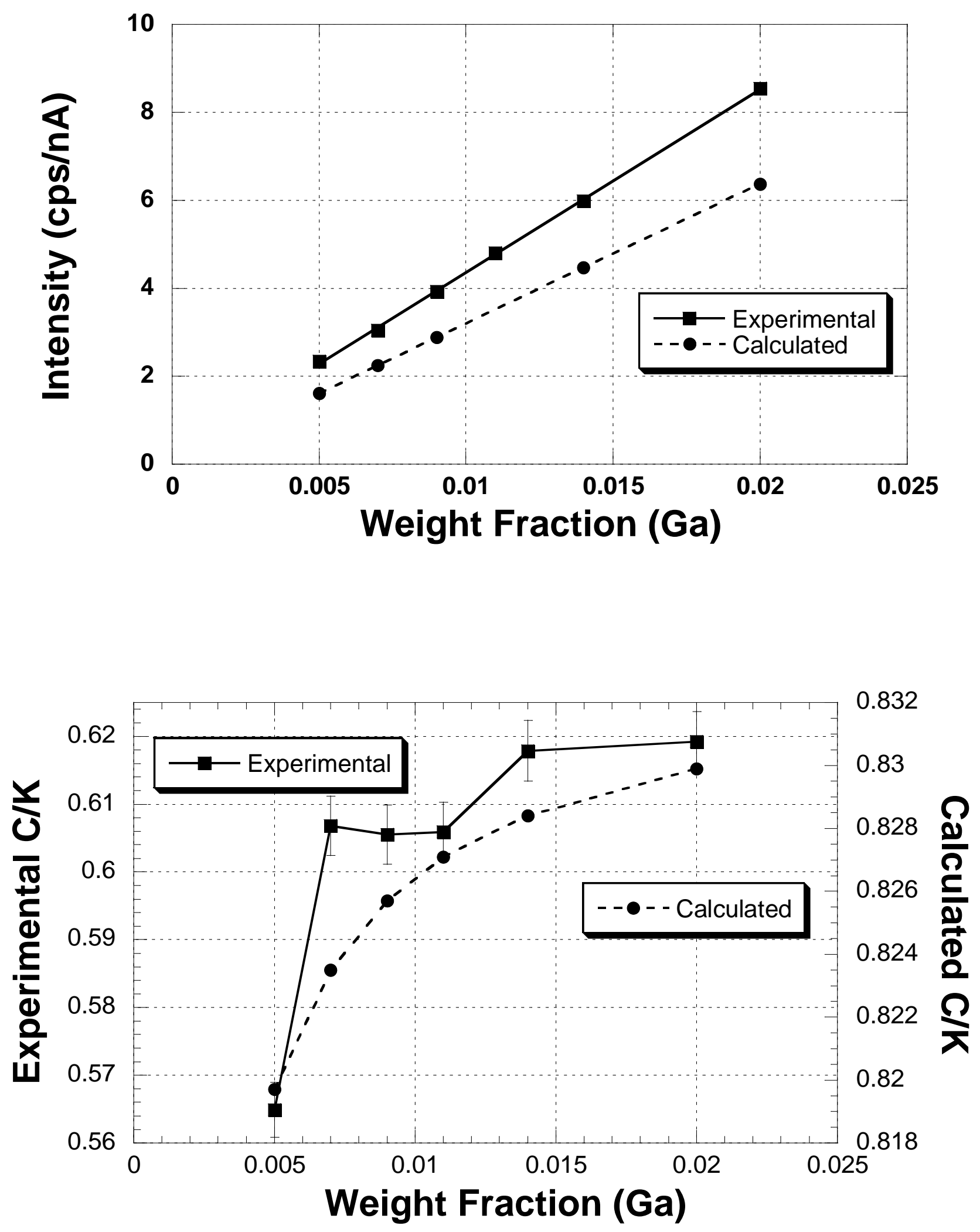